\title{
Vulnerabilidad al cambio climático: Dificultades en el uso de indicadores en dos cuencas de Colombia y Argentina
}

Vulnerability to climate change: Difficulties in using indicators
in two basins of Argentina and Colombia

Paula Mussetta

Coordinadora equipo argentino del proyecto: VACEA

María Julia Barrientos

Coordinadora equipo argentino del proyecto: VACEA

ERIKa Acevedo

Universidad de Antioquia

SANDra TurbaY

Universidad de Antioquia

Olga Ocampo

Universidad Autónoma de Manizales

pmussetta@mendoza-conicet.gob.ar (ARGENTINA)

Recibido: 08.072015

Aceptado: 28.11 .2016

\section{RESUMEN}

Este artículo evidencia las dificultades metodológicas y teóricas en el uso de indicadores sociales para evaluar la vulnerabilidad al cambio climático y de esta forma contribuye a refinar las estrategias metodológicas para el abordaje de este fenómeno. La mayoría de los estudios de vulnerabilidad al cambio climático la definen como una expresión de tres dimensiones: la exposición de los sistemas a los fenómenos climáticos, la sensibilidad a esas condiciones riesgosas, y su capacidad para afrontar los impactos que los afectan. Estas tres dimensiones facilitan la operacionalización del concepto a partir de indicadores biofísicos y sociales cuya principal finalidad es cuantificar el fenómeno y establecer relaciones de 
causalidad entre eventos y recursos. Frente a esta conceptualización, otra manera de entender el fenónemo es definirla como un proceso dinámico determinado procesos sociales, económicos, políticos, culturales e institucionales. Desde este enfoque el cambio climático impacta sobre una serie de vulnerabilidades previas y de largo plazo definidas principalmente por el acceso a los recursos. La vulnerabilidad se define entonces, como un proceso cuya complejidad no admite cuantificaciones ni generalizaciones entre causas y efectos como las que proponen los sistemas de indicadores. A partir de una experiencia de investigación concreta en las cuencas de los ríos Mendoza, en Argentina, y Chinchiná, en Colombia este artículo demuestra las limitaciones que el uso de indicadores de vulnerabilidad social conllevan para la investigación social aplicada. Con base en el análisis de datos estadísticos de uso público disponible en Argentina y en Colombia y de entrevistas en profundidad en las areas de estudio, los autores reconstruyen las dificultades enfrentadas al momento de construir dichos indicadores para demostrar las debilidades de cada indicador para dar cuenta del complejo fenómeno de la vulnerabilidad. Se analizan en particular los indicadores de nivel de ingresos, tenencia de la tierra, acceso al agua, infraestructura tecnológica, asistencia técnica, asociativismo y nivel educativo. Para finalizar, se presenta una propuesta alternativa para dar cuenta de las complejidades propias del fenómeno de la vulnerabilidad.

\title{
PALABRAS CLAVE
}

\begin{abstract}
Vulnerabilidad - cambio climático - indicadores sociales - Colombia - Argentina.
\end{abstract}

\section{ABSTRACT}

This article meets methodological and theoretical difficulties in using social indicators to assess vulnerability to climate change and contributes to enhance methodological strategies for addressing this phenomenon. Most of the studies of vulnerability to climate change define vulnerability as an expression of three dimensions: the exposures of the systems to climatic events, the sensitivity to these risk conditions and the adaptive capacity to cope with the impacts. These three dimensions enable the operationalization of the concept trough biophysical and social indicators whose main purpose is to quantify the phenomenon and establish causal relationships between events and resources. From a different wiew of this conceptualization, another way of understanding vulnerability is to understand it as a dynamic process strongly shaped by social, cultural, economic, political and institutional conditions. From this approach, climate change is suposed to impact on previous and long-term vulnerabilities mainly defined by access to resources. Vulnerability is then defined as a complex process which does not support quantifications or generalizations between causes and effects 
as those proposed in indicators systems. Based upon a research experience in Mendoza, Argentina, and Chinchiná, in Colombia this article demonstrates the limitations of the use of indicators of social vulnerability to applied social research. Through the analysis of public statistical data in Argentina and Colombia and in-depth interviews in the study areas, the authors reconstruct the difficulties faced in the building process of these indicators to demonstrate the weaknesses of each indicator to account the complexity of vulnerability. Income, land tenure, access to water, technology, infrastructure, technical assistance, education and associativism indicators particularly discussed. At the end, the authors presents an alternative proposal to adress the complexities of vulnerability.

\section{KEY WORDS}

$$
\text { Vulnerability - climate change - social indicators - Colombia - Argentina }
$$

\section{INTRODUCCIÓN}

Este artículo aporta evidencia sobre las dificultades en el uso de indicadores para evaluar la vulnerabilidad al cambio climático. Su objetivo es propiciar una reflexión que conduzca a refinar las metodologías de evaluación de la vulnerabilidad en estudios que integran dimensiones biofísicas y sociales y que comparan resultados de distintos estudios de caso.

Durante mucho tiempo, el estudio de los impactos del cambio climático se mantuvo en el ámbito de las ciencias naturales. ${ }^{1}$ Sólo recientemente, el campo fue cediendo terreno a otras disciplinas que sumaron a lo estrictamente físico-climático, dimensiones políticas, sociales, económicas, culturales. A partir de esto, se ha ido generalizando un consenso en la comunidad científica que sostiene que la vulnerabilidad a los cambios y variaciones del clima tiene múltiples determinaciones. Es decir, que está condicionada por factores físicos y sociales y que, por lo tanto, es necesario desarrollar abordajes interdisciplinarios.

La investigación sobre los impactos del cambio climático ha mostrado un giro contundente hacia lo social. No obstante, estos estudios suelen utilizar construcciones teóricas, conceptos y categorías adaptadas de la ecología en particular y de las ciencias naturales en general. Las investigaciones que

\footnotetext{
${ }^{1}$ Cambio climático, Variabilidad Climática y Eventos Extremos son conceptos que se refieren a procesos diferentes. La Variabilidad Climática es la fluctuación del clima por encima o por debajo de un valor medio a largo plazo y se diferencia del Cambio Climático, en tanto éste se refiere a un cambio continuo a largo plazo (aumento o disminución) de las condiciones climáticas. Asimismo, la variabilidad de las temperaturas no siempre se refleja en los valores medios pero sí puede explicar fenómenos puntuales (Eventos Extremos) como heladas inesperadas o picos de temperaturas y precipitaciones que provocan serios daños. No obstante, las diferencias específicas entre estos concpetos no resultan significativas a los fines de este trabajo. En la medida que el trabajo busca evaluar el concepto de vulnerabildad, las particularidades de los conceptos anteriores no modifican la interpretación del análisis que aquí se presenta.
} 
abordan el problema desde categorías propias de las ciencias sociales son aún incipientes. Asimismo, los enfoques y métodos utilizados tienden a reproducir una tradición positivista y las dimensiones sociales se incorporan a los estudios de los impactos del cambio climático en su versión más instrumental, sin llegar a replicar los modelos de generación de conocimiento de las ciencias exactas y naturales.

En esta línea, los indicadores de vulnerabilidad han sido un camino ampliamente utilizado para evaluar los efectos del cambio climático sobre los grupos sociales. El propósito de los indicadores es cuantificar la vulnerabilidad con miras a fortalecer las capacidades para la adaptación, hacer más eficiente la gestión del riesgo y asignar recursos a las poblaciones, localidades, regiones o países que más lo necesitan.

Un aspecto insuficientemente tenido en cuenta es que el uso de indicadores, no sólo prioriza la producción de un tipo de conocimiento sino que también respalda ciertas respuestas políticas al cambio climático (Demeritt 2001; Forsyth 2003).

Las investigaciones que evalúan la vulnerabilidad a partir de indicadores buscan establecer relaciones de causalidad entre eventos (Pahl Wostl et al 2013), cuantificar las dimensiones sociales del problema y establecer generalizaciones que conduzcan la política pública. Pero dicen poco acerca de los procesos sociales y de poder que median la vulnerabilidad (Birkenholtz 2011).

La vulnerabilidad es una condición a priori de las comunidades y de los hogares, resultado de dinámicas sociales, educativas, ecológicas, económicas y políticas subyacentes (Dietz 2013; Narayan y Sahu 2016). Se encuentra vinculada a privaciones existentes más que a amenazas futuras, determinada por un acceso desigual a los recursos y no exclusivamente por los impactos de eventos naturales (Mc Carthy et al 2001; Meza et al. 2010). En este sentido, los determinantes de la vulnerabilidad están necesariamente vinculados a los patrones de desarrollo implementados en los países y regiones (Sarmiento 2008). En esta mirada más compleja, los aspectos climáticos no son entendidos como la causa de la vulnerabilidad sino como factores multiplicadores de vulnerabilidades previas (Montaña 2008). Estos procesos que subyacen a la vulnerabilidad son dinámicos, se manifiestan de manera diferencial en distintas escalas y sectores y son difícilmente cuantificables. A causa de esta complejidad, los sistemas que se basan en la agregación de indicadores estáticos de condiciones locales resultan insuficientes para la evaluación de la vulnerabilidad (Eriksen y Kelly 2007).

A partir de una experiencia de investigación concreta, este artículo demuestra las limitaciones que el uso de indicadores de vulnerabilidad social conllevan para la investigación social aplicada. El trabajo se basa en un estudio, Vulnerabilidad y Adaptación a extremos climáticos en las Américas (VACEA), ${ }^{2}$

2 Programa de Investigación financiado por el Centro de Investigación Internacional para el Desarrollo, el Instituto Canadiense para Investigación en Salud y el Consejo Canadiense de Investigación en Ingeniería y Ciencias Naturales. http://www.parc.ca/vacea/ 
que implicó la construcción de indicadores para las cuencas hidrográficas de los Ríos Mendoza en Argentina y Chinchiná en Colombia. ${ }^{3}$

El río Mendoza se localiza en los Andes Centrales, en la Provincia de Mendoza, en el centro oeste de Argentina (Figura 1). En la cuenca coexisten oasis artificiales de riego con extensos desiertos (Montaña 2008). Los oasis concentran, en un 5\% del territorio provincial, el 97\% de la población (1.686.761 habitantes) y sus actividades económicas (SIAT 2013) exhibiendo un intenso aprovechamiento del agua; mientras que en las zonas sin riego sólo es posible desarrollar cría extensiva de ganado caprino y actividades de subsistencia. La cuenca del río Chinchiná está localizada en el Departamento de Caldas, Colombia, sobre la vertiente occidental de la Cordillera Central de los Andes (Figura 2). Con una superficie de $1052 \mathrm{~km} 2$, concentra el 55\% de la población (53.807 habitantes) y el $80 \%$ de la producción del Departamento de Caldas (Ocampo, Vélez y Londoño 2014). En su territorio se encuentran los municipios de Manizales, Villamaría, Neira, Palestina y Chinchiná cuya población rural se dedica mayoritariamente al cultivo del café.

3 La selección de los dos casos de estudio no se realizó con fines comparativos sino ilustrativos de la inconsistencia de los indicadores. El aspecto de la comparación es tratado en este trabajo como una de las limitaciones de la metodología de indicadores. 
Figura 1: Localización de la cuenca del Río Chinchiná y del Río Mendoza.
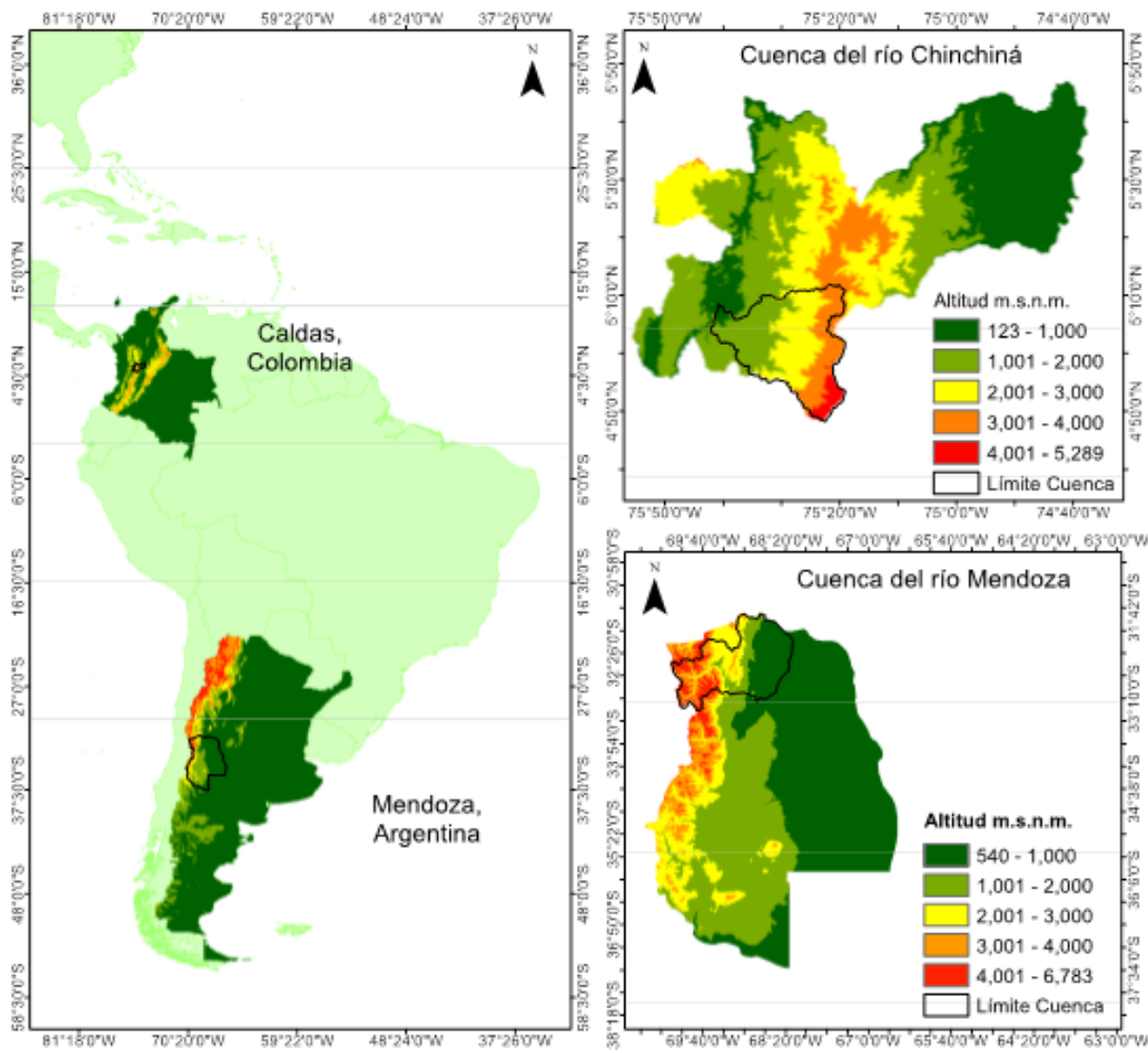

El artículo se organiza en cinco secciones. La primera describe el concepto de vulnerabilidad tal como es utilizado en la investigación sobre cambio y variabilidad climática basados en los desarrollos del IPCC. La segunda da cuenta del uso de indicadores en los estudios sobre la vulnerabilidad. La tercera desarrolla el marco metodológico adoptado por el programa de investigación del que surgen los indicadores trabajados en este artículo. En la cuarta se analizan las dificultades encontradas en la construcción de los indicadores seleccionados y, en la quinta sección esboza una propuesta que aborda la vulnerabilidad desde un enfoque cualitativo y que pretender superar los problemas de los indicadores. Finalmente, la conclusión señala algunas implicaciones del análisis desarrollado en este trabajo. 


\section{LAS TRES DIMENSIONES DEL CONCEPTO DE VULNERABILIDAD.}

Aunque el concepto de vulnerabilidad ha sido utilizado desde hace muchos años en los estudios de pobreza y desarrollo (UNDHA 1992; Brooks 2003; Cardona et al. 2012), su uso más reciente en la investigación sobre cambio y variabilidad climática ha estado influenciado por los sucesivos informes del Panel Intergubernamental para el Cambio Climático (IPCC). En el Tercer Informe (2001), el IPCC, define la vulnerabilidad a partir de una dimensión externa al sistema -expresada en la exposición a los impactos climáticos-, y una interna -referida a la sensibilidad y a la capacidad adaptativa frente a esos factores de estrés externos (Füssel y Klein 2006). En el Cuarto Informe (2007), desplaza la atención hacia aspectos más prácticos de la adaptación, tales como gobernanza, financiamiento, capacidades y transferencia tecnológica (Eakin y Patt 2011). Algunos autores reconocen en este Cuarto Informe un giro desde un análisis de la vulnerabilidad y la adaptación casi exclusivamente cuantitativo y a escalas nacionales, hacia análisis cualitativos e interdisciplinarios orientados a la construcción de capacidades locales y regionales (Brown y Westaway 2011). En el Quinto Informe (2014) queda plasmado que la vulnerabilidad no deriva exclusivamente de factores climáticos sino que es producto de la intersección de procesos sociales expresados en desigualdades socioeconómicas. Esta mirada más amplia y crítica de la vulnerabilidad consolida lo que otros enfoques ya venían sosteniendo desde otras posiciones no dominantes.

Las sucesivas definificiones del IPCC llevan considerar la vulnerabilidad como una función de tres grandes dimensiones: la exposición a la variabilidad y a los extremos climáticos, la sensibilidad de los sistemas sociales y la capacidad de adaptación de los grupos humanos que habitan en las regiones estudiadas.

En las primeras conceptualizaciones del IPCC, la exposición se refiere a los eventos físicos y meteorológicos asociados con la variabilidad climática, a los que un sistema se exhibe, se enfrenta y se "expone" (IPCC 2001). La sensibilidad es el grado en que un sistema resulta afectado por los eventos a los que está expuesto. Los efectos pueden ser directos como un cambio en el rendimiento de los cultivos, o indirectos como daños causados por una mayor frecuencia de inundaciones costeras debido al aumento en el nivel del mar (IPCC 2007). La sensibilidad refleja condiciones sociales, económicas, culturales, políticas y ambientales más amplias y depende de los recursos a los que los actores puedan acceder. Dada la dificultad de separar conceptualmente exposición de sensibilidad, la noción de dobles exposiciones (Leichenko y Obrien 2000) conjuga lo físico y lo social en una misma idea. Al mismo tiempo, como ya mencionamos, el último informe del IPCC (2014) reelabora la noción de exposición incluyendo también dimensiones sociales, de infraestructura y recursos ambientales.

La capacidad de adaptación indica la habilidad de los sistemas para ajustarse a las condiciones del entorno y aumentar su rango de tolerancia 
ante aquellos factores que lo afectan (Díaz y Hurlbert 2012). Se refiere a las características que dan flexibilidad a un sistema para desarrollar estrategias de gestión de riesgos, evitando el aumento de su vulnerabilidad y proveyéndole suficiente grado de libertad para afrontar contingencias futuras (Eakin y Lemos 2006). Los factores determinantes de la sensibilidad coinciden en gran medida con aquellos que limitan la capacidad de adaptación.

Estas tres dimensiones de la vulnerabilidad facilitan la operacionalización del concepto y sobre ellas se erigen muchos de los estudios que utilizan indicadores para evaluar la vulnerabilidad al cambio y la variabilidad climática. Los indicadores analizados en este trabajo también se desprenden de estas dimensiones.

\section{EL USO DE INDICADORES EN EL ESTUDIO DE LA VULNERABILIDAD.}

La construcción de indicadores e índices ha sido ampliamente utilizada para cuantificar la vulnerabilidad y realizar comparaciones entre regiones. Algunas experiencias conjugan las tres dimensiones en un solo índice (Dong et al. 2015), elaboran un índice de alguna de ellas (Acosta et al. 2013) o sintetizan varios índices en uno (Dunford et al. 2015). Para muchos autores, los indicadores resultan pertinentes para realizar amplias comparaciones y para informar decisiones sobre la financiación de la adaptación -aunque sólo sea clasificando regiones en categorías discretas y asumiendo la pérdida de información detallada-(Füssel y Klein 2006).

No obstante, en muchas de estas investigaciones, los autores realizan balances que permiten enumerar algunos obstáculos en el uso de indicadores para medir la vulnerabilidad (Adger 2006; Ahsan y Warner 2014). Una debilidad ampliamente señalada es la de simplificar sistemas complejos como el cambio climático, de variables múltiples, fronteras porosas y lazos imprecisos (Hinkel 2011; Adger 2006); las evaluaciones monodisciplinares son criticadas porque tienden a seleccionar factores obviando los fenómenos abordados por otras disciplinas; además, los que proponen enfoques integrados, carecen de métodos para combinar los distintos tipos de indicadores, o para evaluar su interacción (Nazari, et al. 2015). Usualmente, se requiere una gran cantidad de indicadores para dar cuenta de un fenómeno en forma satisfactoria, pero su número no puede exceder cierto límite porque los modelos se vuelven inmanejables.

Otra preocupación recurrente es la escala de los modelos empleados. Así, los análisis a escala regional o nacional no evidencian vulnerabilidades locales (Krishnamurthy, Lewis y Choularton 2014); de esta forma los problemas de los países en desarrollo requieren de una reducción de escala para poder representar situaciones locales. Otro problema en relación a la escala es que los límites político-administrativos no coinciden con los de la unidad de análisis privilegiada en estos estudios: la cuenca hidrográfica, estimada por el sistema de drenaje natural. 
La posibilidad de medir la vulnerabilidad es una dificultad ampliamente mencionada y se la asocia a problemas como la combinación de datos cualitativos y cuantitativos (Thomas Bohórquez 2013); al carácter dinámico de los procesos biofísicos y sociales que dificulta predecir cambios en las variables relevantes y las leyes que las vinculan (Stern et al. 1992); a los criterios para ponderar las dimensiones analizadas debido a la importancia relativa de los componentes en cada región estudiada (Ashan y Warner 2014); y a los mecanismos disponibles para definir umbrales de vulnerabilidad que reflejen apropiadamente su relatividad, severidad y distribución (Adger 2006). En la base de estas limitaciones, el principal reparo a los sistemas de indicadores como herramienta para estudiar la vulnerabilidad es su incapacidad para dar cuenta de los procesos que dan forma y complejizan el fenómeno.

La revisión de bibliografía especializada nos lleva a sostener que no existen hasta ahora guías para evaluar la vulnerabilidad con base en indicadores que sean universales. La vulnerabilidad, como sostiene Hinkel (2011), es un concepto que puede volverse operativo a través de variables seleccionadas a partir de un modelo teórico o desde métodos estadísticos; lo importante es que se expliquen los criterios y los propósitos con los cuales se construyeron los indicadores.

\section{EL MARCO METODOLÓGICO DEL PROYECTO VACEA.}

Esta sección describe el marco metodológico del estudio en donde se originan los indicadores que analizaremos más adelante. Desde un enfoque integrador e interdisciplinario el proyecto VACEA plantea un modelo de evaluación de la vulnerabilidad en contextos rurales en tres fases (Figura 3). Fase 1: Evaluación de vulnerabilidades pasadas, presentes y futuras, del clima, la agricultura y los recursos naturales a partir de escenarios, modelos y pronósticos. ${ }^{4}$ Fase 2: Análisis de las vulnerabilidades de las comunidades, complementando el uso de datos socioeconómicos secundarios con entrevistas sobre las exposiciones a los riesgos y las sensibilidades y estrategias de adaptación de productores rurales. Esta etapa incluyó también un estudio sobre el papel de las instituciones en la adaptabilidad (gobernanza adaptativa). Fase 3: Desarrollo de indicadores de vulnerabilidad para construir un índice integrando los dos primeros planos (Díaz y Hurberlt 2012). En esta tercera fase, los indicadores para las dimensiones de la vulnerabilidad se definieron a partir de la literatura y de los resultados de las entrevistas realizadas en la fase 1 (Marchildon y Fletcher 2014).

\footnotetext{
${ }^{4}$ En la Fase 1 se procesaron datos exclusivamente biofísicos (hidroclimáticos) y se elaboraron modelos para construir escenarios probabilísticos de escasez de agua en el futuro y eventos climáticos extremos. A partir de estos resultados se construyeron los indicadores de las exposiciones climáticas. Por tratarse de datos y métodos ajenos a las ciencias sociales, este artículo no aborda esta fase del proyecto VACEA.
} 
Figura 2: Modelo metodológico del proyecto VACEA.

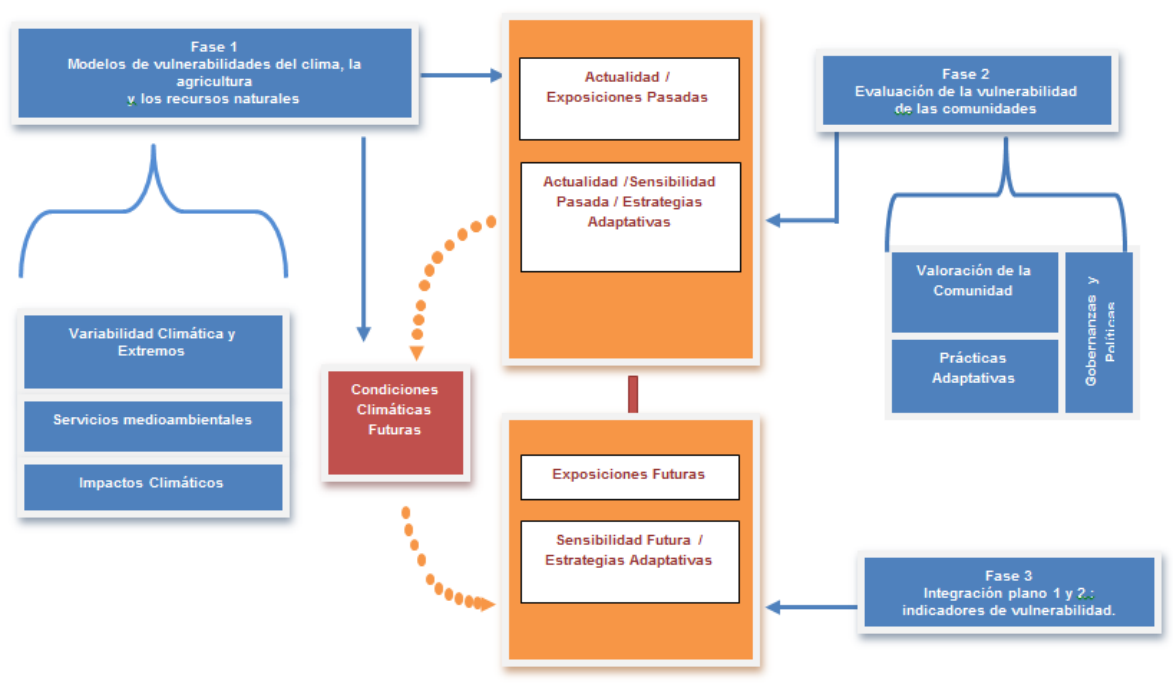

Fuente: Elaboración propia en base a Díaz y Hurlbert 2012.

Previo al desarrollo de las entrevistas, en una etapa previa, se revisaron fuentes secundarias y se realizaron grupos focales con informantes clave que ofrecieron información general sobre el problema de investigación. Estas reuniones a su vez permitieron establecer lazos iniciales, refinar los instrumentos de recolección de información y definir cuáles serían los individuos y las organizaciones consultadas durante la evaluación de la vulnerabilidad de las comunidades rurales para la fase 2 .

En un segundo momento se realizaron entrevistas semi-estructuradas a pobladores rurales, a funcionarios públicos y a representantes de empresas privadas que estuvieran relacionadas con procesos de gobernanza frente a la variabilidad climática. ${ }^{5}$ Las entrevistas a los productores estuvieron orientadas por un conjunto de tópicos abiertos que indagaron sobre las distintas dimensiones de la vulnerabilidad: exposiciones (climáticas y no climáticas), capacidades de adaptación traducidas en conocimientos, prácticas, saberes y las percepciones de los actores sobre el futuro. Las entrevistas a los funcionarios públicos exploraron las dimensiones de la gobernanza adaptativa, entre ellas, la capacidad de respuesta apropiada y oportuna ante los eventos clímáticos, disposición para aprender de experiencias pasadas y modificar las prácticas para favorecer la adaptación ante eventos inesperados, acceso a la información

${ }^{5}$ Las entrevistas se desarrollaron hasta alcanzar la saturación teórica en la que nuevas observaciones ya no conducían a información adicional. 
científica, equidad en la participación de distintos actores, transparencia en la rendición de cuentas, etc. (Díaz y Hurlbert 2012).

En el caso de Colombia se hicieron 5 grupos focales, 30 entrevistas a pobladores rurales y 22 entrevistas a funcionarios y líderes relacionados con procesos de gobernanza. En Argentina, se realizaron 2 grupos focales, 41 entrevistas a productores ${ }^{6}$ a lo largo de los distritos de la cuenca y 25 entrevistas a representantes de instituciones implicadas en la gestión del agua, el medioambiente y la producción agrícola en nivel nacional, provincial y local. Todas las entrevistas fueron grabadas, transcritas y codificadas ${ }^{7}$ antes de establecer interpretaciones de las respuestas comparando los diferentes perfiles de entrevistados.

Una vez concluido el análisis de las entrevistas correspondiente a la segunda etapa del proyecto, se escogieron los indicadores de exposición, de sensibilidad y de adaptación (tabla 1) para completar la etapa 3. Los indicadores seleccionados fueron discutidos con los representantes del gobierno y de las organizaciones locales, con el fin de validar el análisis y redefinirlos, en caso de ser necesario.

\section{ANÁLISIS DE LOS INDICADORES EN LOS DOS CASOS ESTUDIADOS.}

Como se explicó más arriba, la vulnerabildad se define a partir de tres dimensiones la exposición, la sensibilidad y la capacidad adaptativa de las poblaciones ante los eventos climáticos extremos (IPCC 2001). Para el análisis de la exposición se seleccionaron exclusivamente indicadores biofísicos y naturales relativos al clima, el suelo y los cultivos, ${ }^{8}$ estimados a partir largas series de datos cuantitativos (entre 40 y 100 años). En cambio, para las otras dos componentes de la vulnerabilidad se seleccionaron indicadores sociales, que se presentan en la tabla 1, con su respectiva justificación conceptual.

${ }^{6}$ Se consideraron distintos perfiles de productores teniendo en cuenta las siguientes variables: departamento donde se ubica la finca; tipo de actividad productiva (fruti-hortícola, vitícola, mixta); tamaño de las explotaciones (pequeño- hasta 5 ha-, mediano de 5,1 a 25 ha, grande de 25,1- 100 ha)

${ }^{7}$ El análisis de material obtenido en campo se realizó con el software de análisis de datos cualitativos Nvivo 10. Para codificar la información de las entrevistas se definieron nodos, subnodos y atributos (en forma jerárquica, como "árboles") para cada uno de los componentes de la vulnerabilidad. La estructura de codificación se correspondió con las categorías de los cuestionarios de las entrevistas. Para las entrevistas a productores: vulnerabilidades/sensibilidades, estrategias adaptativas y vulnerabilidades futuras para el caso de las entrevistas a productores. Para las entrevistas a actores institucionales: roles institucionales, administración de la organización, flexibilidad, planeación, toma de decisiones y funcionamiento institucional, recursos institucionales y limitaciones, actores que respaldan la institución, rendición de cuentas, redes institucionales.

${ }^{8}$ Promedio anual de precipitaciones, índice de precipitaciones y evapotranspiración estandarizado (SPEI), frecuencia de eventos climáticos extremos y volúmenes de producción. Ver nota 4. 
Tabla 1: Listado completo de indicadores seleccionados para la sensibilidad y la capacidad adaptativa.

\begin{tabular}{|c|c|}
\hline $\begin{array}{l}\text { Indicadores } \\
\text { de } \\
\text { Sensibilidad }\end{array}$ & $\begin{array}{l}\text { Nivel relativo de ingreso: ingresos relativos más bajos impiden } \\
\text { ahorrar para momentos de crisis e inhabilitan a las familias para } \\
\text { introducir innovaciones tecnológicas para adaptarse a los extremos } \\
\text { climáticos. }\end{array}$ \\
\hline & $\begin{array}{l}\text { Tenencia de la tierra: mayores costos de producción para quienes } \\
\text { alquilan la tierra para producir, incapacidad para acceder a créditos, } \\
\text { inseguridad económica por depender de empleos temporales en } \\
\text { predios ajenos. }\end{array}$ \\
\hline & $\begin{array}{l}\text { Acceso al agua potable y para riego: el estrés hídrico empeora las } \\
\text { condiciones del productor rural en caso de una sequía. }\end{array}$ \\
\hline & $\begin{array}{l}\text { Cantidad de hogares y fincas ubicadas en áreas de inundaciones o } \\
\text { deslizamientos: aumenta el riesgo de pérdida de bienes y de vidas } \\
\text { humanas ante precipitaciones intensas. } \\
\text { Índice de Desarrollo Humano: medida resumen de educación, salud } \\
\text { e ingresos. }\end{array}$ \\
\hline $\begin{array}{l}\text { Indicadores } \\
\text { de Capacidad } \\
\text { Adaptativa }\end{array}$ & $\begin{array}{l}\text { Diversificación de ingreso: mejor capacidad de adaptación en } \\
\text { períodos de crisis climáticas en la que los ingresos provenientes de la } \\
\text { producción pueden mermar o desaparecer. }\end{array}$ \\
\hline & $\begin{array}{l}\text { Estado de la infraestructura agrícola: hace más eficiente el uso del } \\
\text { agua y de otros insumos agrícolas, evita pérdidas en la cosecha y } \\
\text { deterioro de la calidad del producto. }\end{array}$ \\
\hline & $\begin{array}{l}\text { Asistencia técnica-científica en problemas agrarios: permite } \\
\text { tomar decisiones anticipadas e introducir cambios en los sistemas } \\
\text { productivos para ajustarse al clima }\end{array}$ \\
\hline & $\begin{array}{l}\text { Nivel de asociativismo: la existencia de redes de apoyo permite a las } \\
\text { familias rurales tener ayuda en momentos de crisis. }\end{array}$ \\
\hline & $\begin{array}{l}\text { Nivel educativo: facilita el acceso a la información técnica } \\
\text { y científica necesaria para prevenir los efectos de los extremos } \\
\text { climáticos sobre los cultivos. También da oportunidades de empleo } \\
\text { en sectores diferentes a los agropecuarios. }\end{array}$ \\
\hline & $\begin{array}{l}\text { Cobertura de salud: El trabajo agrícola exige buenas condiciones } \\
\text { de salud, especialmente cuando se basa en la mano de obra familiar. }\end{array}$ \\
\hline & $\begin{array}{l}\text { Equidad de género: cuando hombres y mujeres tienen iguales } \\
\text { oportunidades, la familia tiene más probabilidad de salir adelante } \\
\text { después de un evento climático extremo pues los dos pueden aportar } \\
\text { en el sostenimiento del hogar y en su recuperación }\end{array}$ \\
\hline & $\begin{array}{l}\text { Nivel de autonomía alimentaria: permite a la familia sobrevivir } \\
\text { cuando se pierde la cosecha del cultivo comercial por factores } \\
\text { climáticos y es especialmente relevante cuando se bloquea el acceso } \\
\text { a centros urbanos por inundaciones o deslizamientos. }\end{array}$ \\
\hline & $\begin{array}{l}\text { Gobernanza: genera capacidad adaptativa por el respaldo } \\
\text { institucional, los sistemas de alerta que evitan desastres, la } \\
\text { oportuna atención si se presentan y da legitimidad a las políticas } \\
\text { de ordenamiento del territorio orientadas a la preservación de los } \\
\text { servicios ambientales delos ecosistemas institucionales. }\end{array}$ \\
\hline
\end{tabular}


Los indicadores más ilustrativos en cuanto a las dificultades metodológicas fueron los relacionados con la economía, el acceso al agua, la infraestructura, la asistencia técnica, el asociativismo y el nivel educativo. Estos son lo que se analizarán a continuación, justificando su inclusión y evaluando su capacidad para dar cuenta de las características de los procesos en cada situación particular.

\subsection{Indicadores de sensibilidad}

\subsubsection{Indicadores económicos e ingresos.}

La economía de una población es, sin lugar a dudas, condicionante de la vulnerabilidad. Aunque el PIB es reconocido como un indicador altamente sensible para capturar esa vulnerabilidad (Gass 2007) contiene varias limitaciones. Por lado, un PIB alto no implica necesariamente menos pobreza. Además, ese nivel puede atribuirse a actividades insostenibles, como la minería, que sólo producen flujos temporales de dinero destruyendo ecosistemas y aumentando la vulnerabilidad de las comunidades. Por otra parte, al ser una medida nacional esconde desigualdades entre regiones y no distingue el peso de los distintos subsectores de la economía -aspecto fundamental para evaluar la vulnerabilidad asociada a sistemas productivos regionales-. Otra limitación del indicador es que el PIB de un solo año no es un reflejo robusto de la situación económica de un país si no se analizan series y tendencias. Por otra parte, puede no ser revelador de un crecimiento económico alto si una gran parte del gasto se destina a atender una guerra interna, como en Colombia. Por lo tanto, debería correlacionarse con las prioridades del gasto público. Por último, es necesario tener en cuenta también la desigualdad en la distribución de la riqueza porque obstaculiza el desarrollo económico y social y genera pobreza.

Ante estas dificultades en el uso exclusivo del PIB como indicador de las condiciones económicas, una alternativa es considerar el nivel de ingresos per cápita. Sin embargo, en este caso sería necesario diferenciar el ingreso per cápita de zonas urbanas y rurales, pues los ingresos de los agricultores siempre son considerablemente más bajos que los que reciben los trabajadores urbanos. El salario mínimo es otra forma para calcular ingresos. Pero para que refleje condiciones económicas debe ser analizado junto con otros factores como el costo de la canasta familiar, el tamaño de los hogares, la tasa de dependencia económica y la estabilidad en la oferta laboral.

Este punto nos lleva a reflexionar, en un paréntesis, sobre la diversificación del ingreso como indicador de las capacidades adaptativas. La literatura científica que aborda la relación entre adaptación al cambio climático y diversificación de ingresos considera que esta última puede reducir la vulnerabilidad a nivel de la unidad doméstica (Miller, Yoon y Yu 2013:11). Sin embargo, las entrevistas nos advierten que quienes tienen tierra y capital suficiente pueden vivir holgadamente de la actividad agrícola. El trabajo asalariado en zonas rurales es ocasional y, si bien permite subsistir tras una mala 
cosecha, suele ser mal pagado. Así, son precisamente los agricultores más pobres quienes tienen ingresos más diversificados. En este sentido, la diversificación no constituye un indicador confiable de la capacidad adaptativa. A veces, evidencia lo opuesto: dificultades para hacer rentable la actividad agrícola, desigualdades sociales y desintegración de la estructura productiva.

La sociologia rural ha realizado aportes en el estudio de la pluriactividad que podrían ser incorporados para fortalecer los indicadores de ingresos de los trabajadores agrícolas (Neiman et al. 2001; Bendini et al. 2003). Este enfoque advierte la necesidad de considerar el origen, los contextos y las funciones de la pluriactividad para detectar si se trata de estrategias de supervivencia de productores en problemas o de acumulación y diversificación en ciclos de prosperidad (Bendini et al. 2003).

Las limitaciones señaladas demuestran que ni el PIB ni el salario son las mejores expresiones de las capacidades económicas. Además, ingreso y vulnerabilidad no siempre están asociados. Entre las opciones para construir indicadores más robustos, emerge el Î́ndice de Necesidades Básicas Insatisfechas (NBI) que aporta información más profunda acerca de los niveles económicos de los hogares (adecuación de la vivienda y sus servicios, dependencia económica, niños no escolarizados). Ahora bien, el NBI tampoco está libre de problemas ya que requiere considerar la escala geográfica por las diferencias entre el país, la región y las subregiones, así como las diferencias entre el sector urbano y el rural. En Mendoza, por ejemplo, en 2010 el nivel de NBI era del 7,6\% un porcentaje inferior al promedio nacional que para ese año era de $9,1 \%$. Pero observando las diferencias entre zonas urbanas y rurales, se advierte que la pobreza rural en Mendoza triplica la pobreza urbana y supera ampliamente el promedio nacional (INDEC 2010).

La revisión de los indicadores usualmente sugeridos para evaluar la vulnerabilidad económica, obliga a ser cautelosos. Cada medida tendrá sus propias limitaciones y esto impide arrojar un argumento conclusivo al respecto. Se evidencia entonces, la necesidad de estar atentos y de buscar opciones adaptadas a la situación que se desea explicar.

\subsubsection{Tenencia de la tierra.}

Los derechos de propiedad, junto con la mano de obra, forman el activo más común para producir alimentos (FAO 2003). Además, influyen en las decisiones de inversión de los agricultores (IFAD 2010), sirven de garantía para acceder a créditos y aumentan la posibilidad de usar nuevas tecnologías y conservar los suelos (Hisali et al. 2011; Beekman y Bulte 2012). Sin embargo, esos derechos no suponen siempre una mejor situación socioeconómica para la familia; eso dependerá del área del terreno, de la calidad de sus suelos, de la disponibilidad de agua, de su cercanía a las carreteras que llevan a los mercados, entre otros factores que lo hacen potencialmente productivo (Montaña 2008).

Puede ser que en una región haya muchos propietarios, pero con minifundios 
que no garantizan ingresos para cubrir las necesidades básicas de la familia. También puede haber muchas compañías multinacionales que producen en terrenos arrendados y que tienen, sin embargo, los conocimientos y los recursos para enfrentar situaciones de stress provocadas por el clima.

La tenencia de la tierra no es suficiente para reducir la vulnerabilidad, si se presentan fenómenos como el desplazamiento por conflictos armados, por problemas ambientales o por la presión de grupos económicos poderosos o si se debilitan las instituciones locales con potencial para reducir la capacidad adaptativa (Fitzpatric 2006, PNUD 2011, Mapfumo et al. 2013).

La concentración de la tierra es uno de los indicadores usualmente empleados para evaluar la vulnerabilidad de la población rural. El coeficiente de Gini mide esa concentración: cuando el valor es más próximo a uno, la posesión de la tierra recae en pocas manos, pero si el valor es cercano a cero, la tierra está distribuida en una mayor cantidad de individuos. En el 2009, el índice Gini de tierras en Colombia fue de 0,86, uno de los más altos en propiedad rural en América Latina y el mundo (PNUD 2011). En el departamento de Caldas, donde se sitúa la cuenca del río Chinchiná, el Gini de tierras era de 0,88 superando el Gini nacional (PNUD 2011). En Argentina el estudio de la FAO (2013) evidencia también procesos de concentración de tenencia e incrementos importantes de propiedad o explotación extranjera.

La propuesta original del proyecto VACEA planteó un indicador de la tenencia definido como el porcentaje de propietarios con respecto al de arrendatarios. En Mendoza, por ejemplo, luego de una reagrupación de los datos disponibles, se observa que el $91 \%$ de los productores rurales son propietarios y el 9\% arrendatarios. Sin embargo, el Censo Nacional Agropecuario (INDEC 2008) define el régimen de tenencia de la tierra en ocho categorías. En consecuencia, la dicotomización de esta variable no deja ver la variedad de condiciones de tenencia que quedan subsumidas bajo la categoría "propietarios". En Colombia, también la complejidad de la tenencia excede la dicotomía propietarios/arrendatarios. Un estudio realizado para la ciudad de Manizales, en la cuenca del río Chinchiná, afirma que el $10 \%$ de los predios corresponde a propiedades colectivas; el 14\% al sistema de aparcería donde el productor cuenta con un acuerdo que le permite explotar la tierra a cambio de un porcentaje de la producción; el $1 \%$ está constituido por colonos que tienen la posesión de sus predios pero no tienen escrituras; el 12\% a arrendatarios, es decir, poseen contrato de arrendamiento formal del predio para explotarlo; y el 62\% a propietarios con escrituras debidamente legalizadas (Gobernación de Caldas 2011). Sin embargo, el $88 \%$ de los propietarios son minifundistas que tienen menos de diez hectáreas (CORPOCALDAS-UNAL 2014).

Adicionalmente, deberían tenerse en cuenta las políticas de ordenamiento territorial y de desarrollo rural, la seguridad, la productividad y la potencial rentabilidad de la tierra y no solamente su propiedad. 


\subsubsection{Acceso al agua.}

El acceso al agua de calidad es uno de los aspectos fundamentales en los estudios sobre la pobreza (Winchester 2008) y un indicador pertinente para evaluar la vulnerabilidad de los hogares. Colombia cuenta con una larga serie de indicadores hidrológicos que permiten evaluar este aspecto de la vulnerabilidad (IDEAM 2010): el índice de regulación hídrica, de aridez, de uso de agua o de escasez, índices de calidad de agua y el índice de vulnerabilidad por desabastecimiento. Pero la abundante disponibilidad de información no contribuye a homogeneizar las diferencias entre las dos cuencas hidrográficas estudiadas aquí, principalmente, debido a las diferencias en el rol del agua en cada una de ellas.

El uso agrícola del agua en la cuenca del Río Chinchiná, no se destina al riego de los cultivos sino a diferentes labores, especialmente lavado del café, previo al secado del grano. Los municipios de la cuenca presentan problemas de calidad en el agua para consumo, en especial en las zonas rurales (INS 2012). El deterioro del recurso obedece al uso de agroquímicos, a la descarga de aguas residuales domésticas e industriales, a la inadecuada disposición de residuos sólidos y a la falta de infraestructura pública e inversión en saneamiento básico (CORPO-CALDAS 2014).

Los indicadores hidrológicos en el Río Chinchiná evidencian excedentes de agua según el índice de aridez; sin embargo, la cuenca tiene bajos índices de retención y regulación hídrica que implican baja capacidad para mantener un régimen de caudales y susceptibilidad a períodos de estiaje, debido a una inadecuada distribución del recurso. Al mismo tiempo, los índices de uso de agua son altos y evidencian estrés hídrico en la cuenca, a pesar de la abundante disponibilidad (Ocampo et al. 2014). Es de anotar también que las marcadas pendientes aumentan el riesgo de deslizamientos y por las características torrenciales de río de montaña, se incrementa la posibilidad de avalanchas.

Mendoza en cambio, es un desierto y el agua cobra un sentido diferente. Si bien el 79,6\% de los hogares rurales tiene acceso a agua potable contra un 99,2\% del área urbana provincial (DEIE 2006), el agua para uso agrícola es la más relevante para estimar la vulnerabilidad al cambio climático. La agricultura está atada a un sistema de riego artificial bajo dos modalidades: agua superficial (proveniente principalmente del deshielo en la cordillera) y subterránea (de pozos y perforaciones en las fincas). Como se dijo al inicio, sólo el 5\% de las tierras de la cuenca están irrigadas (SIAT 2013). El 95\% restante no tiene ni derechos ni infraestructura de riego. Por lo tanto, analizar un indicador de acceso al agua implica, además de evaluar el agua para riego junto a la potable, considerar diferentes aspectos.

En primer lugar, la ubicación del predio. No sólo es relevante si la finca está dentro o fuera de la zona irrigada, sino también, en qué lugar se ubica al interior de los oasis, dado que el agua cuenca arriba es de mayor calidad que la de la parte baja. Además, las fincas más cercanas a la red primaria de distribución tienen más garantías de recibir agua que las que están al final de la cola del 
riego. Adicionalmente, el tipo de licencia de riego implica que los regantes que poseen derechos definitivos reciban un $20 \%$ más de agua que aquellos cuyas tierras tienen un derecho precario. Finalmente, la posibilidad de acceder a agua subterránea como fuente alternativa al riego superficial es fundamental por la alta variabilidad en las dotaciones superficiales (que varían en función de la cantidad de nieve caída en la temporada invernal en la cordillera de Los Andes). Esta es la principal adaptación de los productores, sin embargo, queda restringida a unos pocos por los altos costos que implica el bombeo.

Las realidades de ambas cuencas hidrográficas muestran los problemas de comparabilidad. No podría elaborarse un índice lo suficientemente amplio para expresar las particularidades y a la vez incluir la diversidad de características en torno al uso agrícola. La componente podría mejorarse incluyendo la disponibilidad física de agua; la posibilidad de acceder a ella (para consumo y para riego) incluyendo las disputas por la escasez; las capacidades individuales, comunitarias y de ayuda política para manejar el agua. En este sentido, el Índice de Pobreza Hídrica (Sullivan et al, 2003) incorpora estas subcomponentes que creemos podrían aportar una visión mejorada de la complejidad del acceso al recurso hídrico en las cuencas estudiadas.

\subsection{Indicadores de capacidades adaptativas.}

\subsubsection{Infraestructura climática y asistencia técnica}

Tanto la infraestructura tecnológica como la asistencia técnica científica que reciben los productores son aspectos relevantes para desarrollar estrategias frente a las contingencias climáticas (IPCC 2001 y 2007; Eakin y Lemos 2006). Al mismo tiempo, la cuantificación de ambos procesos no permite comprender su incidencia en la generación de capacidades de adaptación.

En Mendoza, los datos disponibles permitieron valorar el nivel tecnológico alcanzado por los productores (INDEC 2002). Los resultados muestran que el $57 \%$ posee una infraestructura tecnológica precaria para enfrentar las contingencias climáticas y sólo el $14 \%$ de los productores cuentan con equipamiento de alto nivel. Esto, en apariencia, es elocuente para evaluar el estado general de la infraestructura tecnológica en la cuenca. Sin embargo, un indicador de nivel tecnológico más consistente exige evaluar la tecnología según las necesidades de cada cultivo o agricultor en particular, además del estado de esa infraestructura. De esta manera podría evaluarse mejor cuán preparado tecnológicamente se encuentra un productor para afrontar los eventos climáticos. Esta explicación matiza la transparencia de los resultados primeros y dificulta la comparación entre regiones en las que se producen diferentes cultivos.

En la Cuenca del Río Chinchiná se encontraron dificultades para aislar los datos sobre la infraestructura tecnológica asociada a la producción de café debido a que esas instalaciones no son relevadas en los censos. Esto quiere decir que únicamente fue posible acceder a esta información de manera tangencial, 
tras la evaluación de la infraestructura de acuerdo con las variables e indicadores del Índice de Pobreza Multidimensional (IPM) que evalúa el material de los pisos de la vivienda, el material de las paredes y el hacinamiento crítico.

Junto a las debilidades señaladas en cuanto a los datos disponibles, la infraestructura tecnológica como indicador de la capacidad de adaptación en contextos rurales, presenta dificultades en sus supuestos teóricos. Y aquí reside su principal debilidad como indicador de la capacidad de adaptación. Varios estudios respaldan la visión tecnocrática de los procesos de adaptación al cambio climático. De este optimismo tecnólogico (Leach et al. 2012) derivan estrategias socio-tecnológicas que apelan a la capacidad de las ingenierías (Lampis 2013) como el mejor camino para reducir los impactos de las contingencias climáticas (Dietz 2013). Sin embargo, existen alternativas para la construcción de capacidades de adaptación que tienen en cuenta los contextos socio-históricos y visibilizan opciones locales que no necesariamente incluyen la implementación de tecnologías agrícolas o infraestructuras complejas (David et al. 2013).

La tecnología como indicador de la capacidad adaptativa, guarda relación directa con las formas en que se transfiere el conocimiento. En Mendoza, un $72 \%$ de productores recibe asistencia de parte de instituciones privadas (cooperativas, empresas de servicios a la producción e instituciones privadas de fomento a la agroindustria) frente a un $28 \%$ ofrecida por instituciones gubernamentales (nacionales o provinciales) (INDEC 2002). Los datos disponibles permiten identificar los proveedores de asistencia y esto resulta relevante porque es un indicio sobre el costo que genera para los productores. No obstante, no permite conocer dimensiones más complejas como el tipo de asistencia recibida o su pertinencia para las necesidades de los productores, ni tampoco es posible deducir si contribuyen a mejorar o no las capacidades de adaptación de los mismos. Las entrevistas indagaron acerca de la conveniencia de esta asistencia y se encontró que algunos la consideran inútil porque no se realizan "en el campo" (en la finca) sino en lugares externos y porque abordan temas que no siempre son de importancia.

En la cuenca media del Río Chinchiná la asistencia técnica a los productores es prestada por la Federación Nacional de Cafeteros de Colombia (FNCC), siguiendo la figura de la Extensión Rural. Este servicio apunta a "mejorar los métodos y técnicas agrícolas para aumentar la productividad y los ingresos" (FNCC y Cenicafé 2013: 49), lo que lleva a que estrategias de adaptación diferentes a la generación de semillas resistentes a ciertas plagas, estén aún en construcción. Sin embargo, medir la transferencia de conocimiento considerando solamente el apoyo técnico-científico, respalda la idea de que las estrategias de adaptación frente a la variabilidad climática deben provenir de agentes exógenos a las poblaciones rurales y estimula perspectivas asistencialistas que devalúan los conocimientos locales que han mantenido los sistemas agrícolas por miles de años.

Las relaciones sociales y la reproducción de conocimientos y prácticas para la producción cafetera constituyen, por ejemplo, alternativas consistentes para adaptarse a las contingencias. Ello se debe ante todo, a que la caficultura es 
una actividad con un profundo arraigo cultural que ha configurado no solo un paisaje o un sistema económico, sino todo un entramado de saberes, prácticas y relaciones que se convierten en patrimonio de la sociedad, dado que los pequeños productores campesinos se caracterizan por incluir mano de obra familiar de manera intensiva, lo cual, en épocas de crisis, les permite solventar parte de los costos del cultivo, manteniéndose en el negocio. Claramente, para abordar esta otra dimensión de la transferencia de conocimientos un indicador cuantitativo resulta deficiente.

De la misma forma en Mendoza, la contraposición entre tecnología y saberes tradicionales se advierte sólo en las entrevistas cualitativas a los productores. En ellas aparece entre los grandes productores una exaltación de la eficiencia en las prácticas de riego y cultivo. Al mismo tiempo, los pequeños productores reconocen como un problema que el envejecimiento de la población rural debido al éxodo a la ciudad de los jóvenes marca una tendencia cada vez mayor hacia la pérdida de tradiciones, prácticas, conocimientos y saberes construidos por distintas generaciones.

\subsubsection{Asociativismo.}

El capital social se refiere a los recursos vinculados a la posesión de una red duradera de relaciones. El asociativismo, una de las formas más comunes de este capital, genera lazos que facilitan la cooperación y el acceso a oportunidades y recursos. Existe un consenso amplio acerca del papel del asociativismo como determinante de la capacidad de adaptación (Ellis 2000; Lemos 2010), pero no hay criterios unificados sobre la forma de medirlo. Una de las tendencias consiste en cuantificar el número de organizaciones en las que los actores participan. Siguiendo este procedimiento, en Mendoza se advierte un muy bajo nivel de participación ya que más del $90 \%$ de los productores no participa en ninguna asociación (INDEC 2002). En Colombia, no fue posible hallar información similar.

En la búsqueda de indicadores alternativos de capital social, otra manera consiste en evaluar el número de organizaciones existentes en una comunidad determinada y establecer una proporción con el total de población de esa comunidad. A diferencia del primer indicador, éste aporta información sobre el capital social "estructural" existente en una comunidad pero no sobre el uso de ese recurso por parte de los productores.

Una dificultad adicional en la medición del asociativismo es que no todas las asociaciones son igualmente beneficiosas para mejorar la adaptación de los productores a las contingencias climáticas. Es decir, una comunidad con una alta densidad de capital social, leída en cantidad de asociaciones, no aporta conocimiento específico sobre la capacidad de los productores para afrontar los problemas derivados del clima. Un paso importante hacia esta definición implicaría considerar la especialidad de la asociación.

La situación de Mendoza suma otra complejidad al potencial explicativo 
de este indicador. Todos los regantes, sólo por el hecho de acceder al riego, forman parte de las Asambleas de Regantes que constituyen un ícono del sistema de riego local. De esta forma la evaluación del capital social a partir de la participación en esas organizaciones especializadas arrojaría un alto nivel de asociativismo y en consecuencia, una incidencia positiva en las capacidades de adaptación. Sin embargo, a partir de las entrevistas, se ha constatado que los productores no se sienten representados por estas asambleas. Como alternativa, estos mismos productores desarrollan lazos informales de cooperación entre vecinos que resultan mucho más significativos para el desarrollo de capacidades. Para este tipo de capital social no existe un indicador y por ello no podría ser incluido en un índice.

Colombia, muestra un matiz diferente pero igualmente complejo. Allí, por un lado está la FNCC la principal asociación que aglutina a los productores proveyéndoles beneficios. Por el otro lado, están las Juntas de Acción Comunal, fundadas hace cincuenta años para tender puentes entre los campesinos y organizaciones políticas de mayor envergadura. Esas juntas existen legalmente y se renuevan cada año, pero en las entrevistas, sus presidentes reconocen que la participación de la gente es mínima.

La deslegitimación de la participación social en los dos casos vulnera su potencial como indicador de capacidades de adaptación. Al mismo tiempo, los procesos informales que sí contibuyen a fortalecer mecanismos de adaptación, permanecen desdibujados tras una indagación cuantitativa del asociativismo como indicador de la capacidad de adaptación. Ante ello, es importante complejizar los indicadores de asociativismo incluyendo otras aristas como el tipo de asociaciones que agrupan a los productores y de qué manera ellas contribuyen, o no, a fortalecer estrategias de adaptación para problemas del clima, ya sea para incorporar tecnología, fortalecer emprendimientos para acceder a mercados internacionales, posicionarse de manera más competitiva en materia de precios, etc.

\subsubsection{Nivel educativo.}

La educación representa para las comunidades un capital que facilita el acceso a información para enfrentar la variabilidad climática, hacer innovaciones en los sistemas productivos, encontrar fuentes de trabajo alternativas o bien remuneradas y gestionar recursos para proyectos de adaptación, entre otros. Por ello es considerado un buen indicador de la capacidad adaptativa. Sin embargo, en los dos casos estudiados se presentan problemas para comprender, a partir de una estimación cuantitativa, su rol en la formación de capacidades de adaptación.

En Mendoza el $45 \%$ de los hogares rurales de la cuenca tiene un nivel educativo bajo y el 23\% un nivel muy bajo (INDEC 2002). No obstante, a pesar de la disponibilidad de información, más años de escolaridad no implican una estrategia de supervivencia directa para una unidad productiva que se sostiene con mano de obra familiar. Además, es necesario considerar las deficiencias en 
la calidad de la educación en zonas rurales que no prepara a los jóvenes para la agricultura ni aporta herramientas para competir con los jóvenes educados en las ciudades.

Evaluar el mismo indicador en Colombia implicaba acudir al Censo Agropecuario de 1970 (un censo iniciado en 2014 estaba inconcluso al momento de esta investigación). Por esa razón, se exploró la posibilidad de calificar el nivel educativo a través del IDH (Indice de Desarrollo Humano que mide nivel educativo, ingresos y expectativa de vida) y del IPM (Indice de Pobreza Multidimensional que mide condiciones educativas del hogar, condiciones de niñez y juventud, trabajo, salud, servicios públicos domiciliarios y condiciones de la vivienda). Según el IDH, el departamento de Caldas alcanzaba un nivel alto $(0.77$ entre 0 y 1$)$. Analizado este dato en profundidad, se advierte que es el nivel educativo de la capital del departamento el que estaba arrastrando el índice a niveles altos, ocultando las deficiencias educativas de las zonas rurales. El IPM en cambio, permite evidenciar esta diferencia en tanto indica que el 65\% de los hogares del departamento tiene bajo logro educativo, el analfabetismo es del $15,6 \%$, la inasistencia escolar del 7,8\% y el rezago escolar del 27\%. Estas cifras reflejan un panorama muy distinto al del IDH y se ajustan más a la realidad que se percibía durante el trabajo de campo con las familias de los agricultores.

Estimar el nivel educativo presenta dificultades en la comparabilidad de indicadores entre los dos casos y por las diferencias en los resultados arrojados por distintos indicadores dentro de un mismo país. Esto aumenta la debilidad del indicador, para evaluar su impacto en la capacidad de las poblaciones rurales para hacer frente a la variabilidad climática. Entonces, más que emplear un indicador numérico, se requiere combinar variables que permitan aprehender los condicionantes o potencialidades de las comunidades. Un índice de vulnerabilidad debe contribuir a complejizar las situaciones que agudizan la pobreza de las comunidades (Spicker et al. 2009), procurando visualizar todas sus aristas: la pobreza como un concepto material (necesidad, un patrón de privaciones, limitaciones de recursos); como situación económica (nivel de vida, desigualdad, posición económica); en términos de condiciones sociales (clase, dependencia de la asistencia social, carencias de seguridad básicas, ausencia de titularidades, exclusión) y como un juicio moral (definida por condiciones materiales que resultan moralmente inaceptables) .

\section{LINEAMIENTOS PARA UN ESTUDIO DE VULNERABILIDAD COMPLEMENTARIO A LOS INDICADORES.}

La evaluación de la vulnerabilidad requiere algo más que un catálogo de indicadores cuantitativos. Se necesitan herramientas que aborden las causas profundas de la vulnerabilidad, que consideren la perspectiva de los actores sociales implicados y que sean capaces de evidenciar las complejidades de los procesos que dan forma a la misma.

Reducir la vulnerabilidad implica modificar el contexto político, económico, 
social e institucional con el cual interactúan los actores sociales que padecen las exposiciones al cambio climático y a la variabilidad climática (O Brien et al. 2007) y contribuir a que los actores desarrollen capacidades de adaptación. Es necesario, en consecuencia, avanzar en una perspectiva holística que integre el estudio de los procesos estructurales en los que actúa el cambio climático, que identifique las causas de la vulnerabilidad y que desde allí plantee posibles cursos de acción.

Una propuesta metodológica para abordar estos señalamientos es plantear un esquema que identifique los principales nodos críticos actuales de la vulnerabilidad en cada área de estudio y que evalúe los procesos que los determinan y sus consecuencias. ${ }^{9}$ Asimismo que identifique y analice las estrategias de adaptación (presentes y futuras) desarrolladas por los actores sociales e institucionales, evaluando el impacto que esas adaptaciones tienen o tendrían para reproducir o transformar las condiciones de vulnerabilidad. La figura 3 muestra el esquema sugerido y algunas preguntas básicas para guiar el análisis.

\section{Figura 3: Esquema para el análisis de los nodos de vulnerabilidad.}

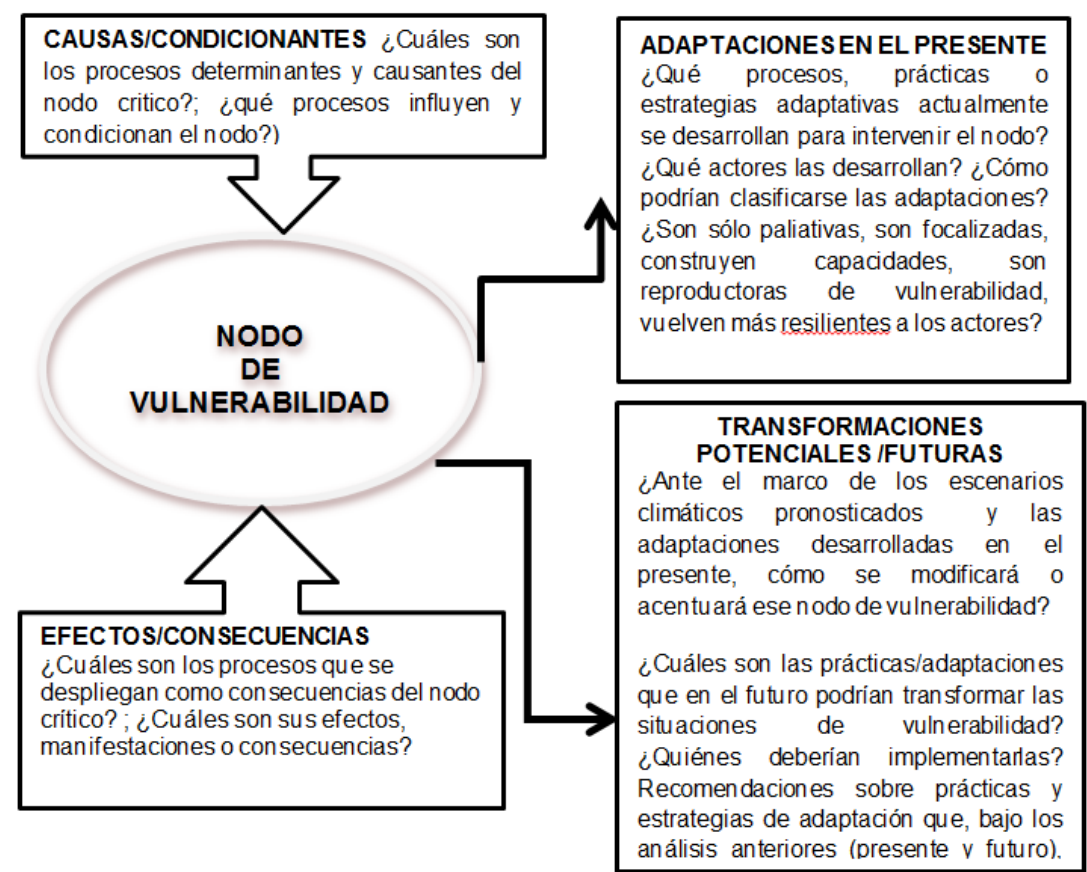

${ }^{9}$ Sin duda el insumo privilegiado para la construcción de los nodos debería ser el de las fuentes primarias recabadas a partir de técnicas cualitativas como entrevistas y grupos focales, combinado con el análisis de fuentes secundarias. 
Los nodos de la vulnerabilidad representan aquellos procesos críticos de orden social, económico, ambiental, agrícola, climático y político que configuran en el presente la vulnerabilidad de las áreas bajo estudio. Ellos facilitan el análisis transversal del problema por medio de enunciados sintéticos que remiten más a una estrategia narrativa que a una orientada a la cuantificación de un fenómeno estático.

Los nodos permitirían así, dar cuenta del carácter dinámico de la vulnerabilidad y evaluar las posibles vulnerabilidades futuras considerando las adaptaciones actuales y los escenarios climáticos pronosticados. Esto es posible gracias a la evaluación de los distintos tipos de estrategias de adaptación incluyendo las preventivas y las reactivas, las espontáneas y las planificadas, las que fortalecen la sostenibilidad y las que destruyen en el largo plazo las bases que garantizan la reproducción de los sistemas sociales y naturales.

Los nodos facilitan también la evaluación de las estrategias con potencial para transformar las condiciones sociales que estructuran la vulnerabilidad. Un esquema como este permite, además, sentar las bases para un proceso de socialización y co-producción de conocimiento entre científicos, funcionarios públicos, productores y otros actores sociales, a partir de espacios de análisis de las capacidades de adaptación actuales.

La siguiente tabla muestra los nodos identificados en las cuencas estudiadas. ${ }^{10}$

\section{Tabla 2: Los nodos de vulnerabilidad en las cuencas estudiadas.}

\begin{tabular}{|c|c|}
\hline \multicolumn{2}{|r|}{ Identificación de nodos de vulnerabilidad en cada una de las cuencas } \\
\hline \multirow[t]{5}{*}{$\begin{array}{l}\text { Río } \\
\text { Chinchiná }\end{array}$} & $\begin{array}{l}\text { Impacto negativo de la variabilidad climática en la productividad } \\
\text { cafetera y probable acentuación de la problemática a causa del cambio } \\
\text { climático en el siglo XXI }\end{array}$ \\
\hline & Gestión ambiental incipiente y prácticas que afectan la sostenibilidad \\
\hline & Riesgos por la exacerbación de eventos hidrometeorológicos extremos \\
\hline & Reconfiguración de las dinámicas agropecuarias de la región \\
\hline & Políticas públicas que desestimulan el sector agropecuario. \\
\hline \multirow{5}{*}{$\begin{array}{c}\text { Río } \\
\text { Mendoza }\end{array}$} & Crisis del sector agrícola por secuelas del clima \\
\hline & Brecha de rentabilidad entre costos de producción/precio de productos \\
\hline & $\begin{array}{l}\text { Hegemonía del paradigma de eficiencia tecnológica por sobre otras } \\
\text { respuestas }\end{array}$ \\
\hline & $\begin{array}{l}\text { Fragmentación institucional en tres planos: } 1 \text {. agua y suelo; } 2 \text {. fuentes } \\
\text { de agua } 3 \text {. oferta y demanda de agua }\end{array}$ \\
\hline & $\begin{array}{l}\text { Problemas de la gobernanza del conocimiento: brecha entre la } \\
\text { generación, movilización y uso de la información sobre clima y agua, } \\
\text { sobre mercados y producción }\end{array}$ \\
\hline
\end{tabular}

10 Por razones de espacio no se incluye el análisis detallado de los nodos, con los determinantes, consecuencias, adaptaciones y transformaciones. No obstante, el mismo podrá ser consultado en un libro que actualmente está en preparación. 


\section{CONCLUSIONES.}

El objetivo de este artículo fue discutir la utilidad de los indicadores para comprender la vulnerabilidad ante el cambio climático. Para ello, se reconstruyó una experiencia de elaboración de indicadores en dos cuencas hidrográficas de Colombia y Argentina, señalando las limitaciones encontradas, y sugiriendo opciones para complejizarlos y contextualizarlos. También se esbozó una propuesta para realizar un análisis cualitativo de la vulnerabilidad de tal forma que pudiera abordarse en términos de procesos complejos y no de relaciones lineales. Para concluir se repasan los principales aprendizajes e interrogantes que este análisis despliega.

Una primera reflexión gira en torno a la capacidad de los indicadores como herramienta explicativa de la vulnerabilidad a eventos climáticos. La complejidad del fenómeno de la vulnerabilidad encuentra explicaciones restringidas si solo es abordada desde indicadores cuantitativos como los aquí presentados, que, por sí solos no reflejan la dinámica social de las comunidades. Por el contrario, homogenizan y simplifican realidades, desdibujando problemas estructurales que definen los territorios y la vulnerabilidad de sus comunidades.

Una segunda reflexión se refiere a la utilidad "social" de los indicadores cuantitativos y nos conduce a preguntar a quiénes y para qué puede servir. De acuerdo con la experiencia reconstruida, el para qué, está ligado a la toma de decisiones sobre financiación internacional y la promoción de políticas de adaptación. En tanto en el para quiénes se ubican los actores gubernamentales y organismos de financiamiento. Ampliar el abanico de interlocutores que usan este tipo de herramientas de evaluación implicará orientar las preguntas hacia otra arena de discusión y cuestionar si pueden servir a la toma de decisiones de otros actores y contribuir a las reivindicaciones de movimientos y organizaciones sociales.

Una tercera reflexión está referida a las políticas inspiradas por estos indicadores de vulnerabilidad pues, usualmente, invisibilizan aspectos estructurales en la distribución de poder como la inequidad y la distribución de recursos. Cabe preguntarse hasta qué punto las políticas derivadas de este tipo de estudios pueden transformar las condiciones de vida de los pueblos ya que no promueven soluciones integrales para enfrentar la vulnerabilidad.

La cuarta y última reflexión se relaciona con el papel de la producción, transferencia y distribución del conocimiento científico. En este sentido, por un lado, es imprescindible abogar a la honestidad científica y remarcar la necesidad de explicitar los supuestos teóricos y epistemológicos de cada decisión. Debe recordarse que los conceptos condicionan no sólo métodos, sino también definiciones políticas sobre la toma de decisiones, la distribución de recursos y la visión sobre los actores sociales. Esto compromete a la comunidad científica en avanzar hacia estrategias interdisciplinarias para el abordaje de problemas socioambientales como el de la vulnerabilidad, procurando trascender los enfoques de una ciencia social positiva y funcional. Sin lugar a dudas, para lograr una estrategia interdisciplinaria, se deberá primero reconocer las 
implicaciones epistemológicas y políticas que esto conlleva; y solo desde aquí, será posible contribuir a una labor académica y socialmente comprometida con el tratamiento de temas tan complejos como los socioambientales.

Sin comprender las causas profundas que explican los procesos de vulnerabilidad es imposible que la comunidad científica brinde herramientas para la toma de decisión política. Incidir y aportar a la transformación de las condiciones sociales de vulnerabilidad de comunidades rurales implica necesariamente contribuir a una redistribución de los recursos y la mejora del acceso a la financiación, la tierra, la tecnología, el agua y otros activos, así como un mayor acceso a la toma de decisiones y la gobernanza.

\section{BIBLIOGRAFÍA}

ACOSTA, L., et al (2013): "A spatially explicit scenario-driven model of adaptive capacity to global change in Europe", Global Environmental Change, 23(5), pp. 1211-1224

ADGER, W.N. (2006): "Vulnerability”, Global Environmental Change, 16 (3), pp. 268-281

ASHAN, N. Y WARNER J., (2014): "The socioeconomic vulnerability index: A pragmatic approach for assessing climate change led risk. A case study in the south -western coastal Bangladesh", International Journal of Disaster Risk Reduction, 8, pp- 32-49

BEEKMAN, G. y BULTE E. H. (2012): "Social norms, tenure security and soil conservation: evidence from Burundi", Agricultural Systems, 108, pp-50-63

BENDINI, M. MURMIS, M. y TSAKOUMAGKOS, P., (2003) El campo en la Sociología actual. Una perspectiva latinoamericana. La Colmena, Buenos Aires, 383

BIRKENHOLTZ, T., (2011) Network political ecology: Method and theory in climate change vulnerability and adaptation research Progress in Human Geography 36(3) pp. 295-315

BROOKS, N. W. (2003): Country level risk measures of climate-related natural disasters and implications for adaptation to climate change, disponible en: http://tyndall.ac.uk/ sites/default/files/wp26.pdf [consulta: 19-6-2015]

BROWN, K. Y WESTAWAY, E., (2011): “Agency, Capacity, and Resilience to Environmental Change: Lessons from Human Development, Well-Being, and Disasters", Annual Review of Environment and Resources, Vol. 36, pp. 321-342

BRUCE, J., WENDLAND, K., NAUGHTON-TREVES, L., (2010): "Whom to Pay? Key Concepts and Terms regarding Tenure and Property Rights in Payment-based Forest Ecosystem Conservation", Land Tenure Center Policy Brief, Land Tenure Center, Madison, WI.

CARDONA, O., ORDAZ, M., YAMIN, L., SINGH, S., BARBAT, A., (2012): "Metodología de Modelación Probabilística de Riesgos Naturales. Modelos de evaluación de amenazas naturales y selección", CAPRA, Probabilistic Risk Assessment Iniciative, ERN Evaluación de riesgos Naturales-América Latina, disponible en http://www.preventionweb.net/english/hyogo/gar/2013/en/bgdocs/ CIMNE\%20et.al.\%202013a-SPA.pdf [consulta: 19-6-2015] 
CEBALLOS, L. y POVEDA, G. (2005): "Predicción de la temperatura superficial del mar en la región Niño 3-4 durante 2002-2003 usando modelación lineal inversa”, Avances en Recursos Hidráulicos, 12, pp. 183-198

CORPO- CALDAS (2014): Síntesis diagnóstica. Plan de Gestión Integral del Recurso Hídrico en la Cuenca del río Chinchiná. Manizales, Universidad Nacional de Colombia

DAVID, A. BRABY, J., ZEIDLER, J., KANDJINGA, L. y NDOKOSHO, J., (2013): "Building adaptive capacity in rural Namibia", International Journal of Climate Change Strategies and Management, 5: 2, pp. 215 - 229

DEIE (2006): Resultados del estudio de condiciones de vida de los hogares rurales y urbanos, Gobierno de Mendoza, disponible en: http://www.deie.mendoza.gov. ar/publicaciones/detalle publicaciones.asp?filtro $=$ Publicaciones + Sociales $\&$ id $=32$ [consulta: 19-6-2015]

DÍAZ H. Y HULBERT, M (2012): "Vulnerability and Adaptation to Climate Extremes in the Americas (VACEA)", Methodology Document for Themes 1A and 1C, No publicado.

DIETZ, K. (2013): “Hacia una teoría crítica de vulnerabilidad y adaptación: aportes para una reconceptualización desde la ecología política”, en: Culturas, conocimientos, políticas y ciudadanías en torno al cambio climático, Bogotá, Biblioteca Abierta Colección General, serie Perspectivas Ambientales, pp. 19-46

DONG, Z., ZHIHUA, P., PINGLI, A., LIWEI, W., JINGTING, Z., DI, H., HUIJIE, H., XUEBIAO, P., (2015): “A novel method for quantitatively evaluating agricultural vulnerability to climate change", Ecological Indicators, 48, pp. 49-54

DUNFORD, R., HARRISON, P., JÄGER, P., ROUNSEVELL, M. Y TINCH, R. (2015): "Exploring climate change vulnerability across sectors and scenarios using indicators of impacts and coping capacity" Climatic Change, 128: 3-4, pp. 339-354

EAKIN, H. Y LEMOS, M.C. (2006): "Adaptation and the state: Latin America and the challenge of capacity-building under globalization", Global Environmental Change, 16, pp. 7-18

EAKIN, H Y PATT, A. (2011) : "Are adaptation studies effective, and what can enhance their practical impact?", WIRES Climate Change, 2, pp: 141-153

ELLIS, F. (2000) : Rural Development and Diversity in Developing Countries, Oxford, Oxford University Press

ERIKSEN, S.H. y KELLY, P.M. (2007): Developing credible vulnerability indicators for climate adaptation policy assessment. Mitigation and Adaptation Strategies for Global Change (2007) 12. pp. 495-524

FAO (2003) : "Tenencia de la tierra y desarrollo rural", disponible en http://www.fao. org/docrep/005/y4307s/y4307s00.htm [consulta: 1-7-2014]

(2013) : "Dinámicas del mercado de la tierra en América Latina y el Caribe : concentración y extranjerización ", disponible en http://www.fao.org/3/contents/ fb3c3698-1aa5-5145-8049-59db19773230/i2547s00.htm [consulta: 15-8-2014]

FITZPATRICK, D. (2006) : "Evolution and chaos in property right systems: the third world tragedy of contested access", Yale Law Journal, 115, pp. 996-1048

FNCC y CENICAFÉ (2013): Manual del Cafetero Colombiano. Investigación y tecnología para la sostenibilidad de la caficultura. Tomo I. Chinchiná, LEGIS

FÜSSEL, H.M Y KLEIN, R. (2006): "Climate change vulnerability assessments an evolution of conceptual thinking", Climate Change, 75: pp. 301-329

GASS, C. (2007): "El informe Stern sobre la Economía del Cambio Climático", Ecosistemas, 16: 1, pp. 124-125 
GOBERNACIÓN DE CALDAS (2011): "Diagnóstico Rural”, Universidad de Caldas, Secretaría de Agricultura de Caldas, disponible en: http://siap.agrocaldas.gov. co/?municipio=centrosur [consulta: 27/03/2014)

HISALI, E., BIRUNGI P., y BUYINZA F. (2011): "Adaptation to climate change in Uganda: Evidence from micro level data", Global Environmental Change, 21 (4), pp. $1245-1261$

HINKEL, J. (2011): "Indicators of vulnerability and adaptive capacity: towards a clarification of the science-policy interface", Global Environmental Change, 21 pp: 198-208 disponible en: http://unfccc.int/resource/docs/natc/colnc1.pdf [consulta: 19-6-2015]

IDEAM (2010): "2a Comunicación Nacional ante la Convención Marco de las Naciones Unidas sobre el Cambio Climático", Bogotá, disponible en: http:// documentacion.ideam.gov.co/openbiblio/bvirtual/021658/2Comunicacion/ IDEAMTOMOIIPreliminares.pdf [consulta: 19-6-2015]

IFAD (2010): "Rural Poverty Report 2011: New realities, new challenges: new opportunities for tomorrow's generation", disponible en http://www.ifad.org/ rpr2011/ [consulta: 19-6-2015]

INDEC (2002): Censo Nacional Agropecuario. Instituto Nacional de Estadísticas y Censos. Ministerio de Economía. Argentina

(2008): Censo Nacional Agropecuario. Instituto Nacional de Estadísticas y Censos. Ministerio de Economía. Argentina.

(2010): Censo Nacional de Población. Instituto Nacional de Estadísticas y Censos. Ministerio de Economía

INS (2012): "Vigilancia de la calidad de agua para consumo humano en Colombia año 2012. Ministerio de Salud y Protección Social", disponible en: http://www.minsalud. gov.co/sites/rid/Lists/BibliotecaDigital/RIDE/IA/INS/Informe\%20Vigilancia\%20 Calidad\%20Agua\%20a\%C3\%B10\%202012[1].pdf [consulta: 1-6-2015]

IPCC (2001): "Climate change 2001: synthesis report. A contribution of Working Groups I, II and III to the Third Assessment Report of the Intergovernmental Panel on Climate change", New York y UK, disponible en: http:77www.grida.no/climate/ ipcc_tar/vol4/Spanish/pdf/front.pdf [consulta: 19-6-2015]

(2007): "Climate Change 2007: Impacts, Adaptation and Vulnerability. Contribution of Working Group II to the Fourth Assessment Report of the Intergovernmental Panel on Climate Change", Cambridge University Press, Cambridge, UK y Nueva York, disponible en: http://www.ipcc.ch/publications_ and_data/ar4/ wg2/en/contents.html [consulta: 19-6-2015]

(2014): "Climate Change 2014: Impacts, Adaptation and Vulnerability. IPCC Working Group II Contribution to AR5", disponible en: http://www. europeansources.info/showDoc?ID=1198539 [consulta: 19-6-2015]

KRISHNAMURTHY, P., LEWIS, K. Y CHOULARTON, R.J. (2014): “A methodological framework for rapidly assessing the impacts of climate risk on national-level food security through a vulnerability index", Global Environmental Change, 25, pp. 121-132

LAMPIS, A. (2013): "Vulnerabilidad y adaptación al cambio climático: debates acerca del concepto de vulnerabilidad y su medición", Cuadernos de Geografía, 22 :2, Bogotá, Universidad Nacional de Colombia, pp. 17-33.

LEACH, M. FAIRHEAD, J., y FRASER, J. (2012): "Green Grabs and Biochar: Revaluing African Soils and Farming in the New Carbon Economy", Journal of Peasant Studies , 39:2, pp: 285-307 
LEICHENKO, R. M. Y O'BRIEN, K. (2000): “Double exposure: Assessing the impacts of climate change within the context of economic globalization", Global Environmental Change, 10, pp. 221-232

MC CARTHY, J., CANZIANI, O., LEARY, N., DOKKEN, D. Y WITHE, K. (2001): Climate change 2001: impacts, adaptation, and vulnerability. Cambridge, Cambridge University Press.

MAPFUMO, P., ADJEI-NSIAH, S., MTAMBANENGWE, F., CHIKOWO, R. Y GILLER, K. (2013): "Participatory action research as an entry point for supporting climate change adaptation by smallholder farmers in Africa", Environmental Development, 5, pp. 6-22.

MARCHILDON, G. Y FLETCHER, A. (2014): "Rural Adaptability to Climate Extremes (RACE) Index: Indicators at Four Levels of Analysis" Working Paper. No publicado

(2014): "Year 2: Integrating the project results", disponible en: http:// www.parc.ca/vacea/assets/PDF/vacae\%20newsletter\%206.pdf [consulta: 19-62015]

MEZA, L., CORSO, S. Y SOZA, S. (2010): Gestión del riesgo de sequía y otros eventos climáticos extremos en Chile. Santiago de Chile, ODEPA/FAO

MILLER, S., YOON, S. Y YU, B. (2013): "Indicators of adaptation to climate change and policy implications for IDB Projects", Inter-American Development Bank, Policy Brief, disponible en: http://www.iadb.org/en/research-and-data/publicationdetails, 3169.html?pub id=IDB-PB-184 [consulta: 19-6-2015]

MONTAÑA, E (2008): "Las disputas territoriales de una sociedad hidráulica. Conflictos en torno al agua en Mendoza, Argentina", Revista Interamericana de Economía Ecológica,

Quito, FLACSO, 9, pp. 1-17

NARAYAN, K. y SAHU, S.K. (2016): "Effect of climate change on household economy and adaptive responses among agricultural households in eastern India" Current Science, vol. 100, N. 7, pp 1240-1250

NAZARI, S., PEZESHKI-RAD, G., SEDIGHIA, H. Y AZADIBET, H. (2015): "Vulnerability of wheat farmers: Toward a conceptual framework", Ecological Indicators, 52, pp. 517-532

NEIMAN, G., BARDOMAS, S. Y JIMENEZ, D. (2001): Entre el campo y la ciudad. Desafíos y estrategias de la pluriactividad en el agro., Ed. Ciccus. Buenos Aires

OCAMPO, O., VELEZ, J. Y LONDOÑO, A. (2014): Análisis de Vulnerabilidad hídrica de la cuenca del río Chinchiná, Manizales, Universidad Nacional de Colombia sede Manizales. Manizales.

PAHL -WOSTL C., GIUPPONI, C., RICHARDS, K., BINDER, C., DE SHERBININ, A., SPRINZ, D., TOONEN, T., VAN BERS, C., (2013): Environmental Science \& Policy 28 pp. 36-47

PNUD (2011): "Colombia rural, razones para la esperanza. Informe Nacional de Desarrollo Humano 2011", Bogotá, disponible en: http://hdr.undp.org/sites/default/ files/nhdr_colombia_2011_es_resumen_low.pdf [consulta: 19-6-2015]

SARMIENTO, J. P. (2008): Gestión del riesgo ante la variabilidad y cambio climático. En: ALDUNCE, P., NERI, C. , SZLAFZTEIN, C. Hacia la evaluación de prácticas de adaptación ante la variabilidad y el cambio climático.Belem, NUMA/UFPA, pp. 31-42

SIAT (2013): disponible en: http://www.siat.mendoza.gov.ar [consulta: 3-7-2014] 
SPICKER, P., ÁlVAREZ, S. Y GORDON, D. (2009): Pobreza. Un glosario internacional, Buenos Aires, CLACSO

STERN, P., YOUNG, O., Y DRUCKMAN, D., (1992): Global Environmental Change: understanding the human dimensions, Washington D.C., National Academy Press

SULLIVAN, C., MEIGH, J.R. y GIACOMELL, A. (2003): "The water poverty index: development and application at the community scale" Natural Resources Forum, 27, pp. $189-199$

THOMAS BOHORQUEZ, J. (2013): "Evaluación de la vulnerabilidad social ante amenazas naturales en Manzanillo (Colima). Un aporte de método", Investigaciones Geográficas, 81, pp. 79-93

UNDHA (1992): "Internationally Agreed Glossary of Basic Terms Related to Disaster Management", Geneva, United Nations Department of Humanitarian Affairs.

WINCHESTER, L. (2008): "La dimension económica de la pobreza y precariedad urbana en las ciudades latinoamericanas. Implicaciones para las políticas de hábitat", Revista Eure, XXXIV:103, pp. 27-47.

\section{SIGLAS.}

FNCC: Federación Nacional de Cafeteros de Colombia PBI: Producto Bruto Interno.

IPCC: Panel Intergubernamental para el Cambio Climático

IPM: Indice de Pobreza Multidimensional.

NBI: Necesidades Básicas insatisfechas.

UNDH: Departamento de Asuntos Humanitarios de las Naciones Unidas. 
